Research in Astron. Astrophys. 201x Vol. X No. XX, 000-000

http://www.raa-journal.org http://www.iop.org/journals/raa

$\boldsymbol{R}$ esearch in

Astronomy and

Astrophysics

\title{
Singlet pairing gaps of neutrons and protons in hyperonic neutron stars
}

\author{
Yan $\mathrm{Xu}^{1}$, Cheng-Zhi Liu ${ }^{1}$, Cun-Bo Fan ${ }^{1}$, Xing-Wei Han ${ }^{1}$, Xiao-Jun Zhang ${ }^{1}$, Ming-Feng \\ $\mathrm{Zhu}^{2}$, Hong-Yan Wang ${ }^{3}$, Guang-Zhou Liu ${ }^{2}$ \\ 1 Changchun Observatory, National Astronomical Observatories, Chinese Academy of Sciences, \\ Changchun 130117, China; \\ Corresponding Author.C.Z.Liu,lcz@cho.ac.cn; Y.Xu,xuy@cho.ac.cn \\ 2 College of Physics, Jilin University, Changchun 130012, China \\ 3 College of Physics, Beihua University, Jilin 132013, China
}

Received 2014 May 26; accepted 2014 July 28

\begin{abstract}
The ${ }^{1} S_{0}$ nucleonic superfluids are investigated within the relativistic mean-field model and Bardeen-Cooper-Schrieffer theory in hyperonic neutron stars. The ${ }^{1} S_{0}$ pairing gaps of neutrons and protons are calculated based on the Reid soft-core interaction as the nucleon-nucleon interaction. We have studied particularly the influence of hyperons degrees of freedom on the ${ }^{1} S_{0}$ nucleonic pairing gap in neutron star matter. It is found that the appearance of hyperons has little impact on baryonic density range and size for the ${ }^{1} S_{0}$ neutronic pairing gap, the ${ }^{1} S_{0}$ protonic pairing gap also decreases slightly in this region $\rho_{B}=0.0-0.393 \mathrm{fm}^{-3}$. However, if baryonic density becomes greater than $0.393 \mathrm{fm}^{-3}$, the ${ }^{1} S_{0}$ protonic pairing gap obviously increases. In addition, the protonic superfluid range is obviously enlarged due to the presence of hyperons. In our results, the hyperons change the ${ }^{1} S_{0}$ protonic pairing gap which must change the cooling properties of neutron stars.
\end{abstract}

Key words: dense matter — (stars:) pulsars: general — equation of state

\section{INTRODUCTION}

In resent years, neutron stars(NSs) have been becoming one of the hottest scientific problems in the domain of astrophysics. The reasoning is that the nucleonic energy gap and corresponding superfluidity(SF) critical temperature can greatly affect the neutrino emission which dominated about $10^{5}-10^{6}$ years of NS cooling phases(Zuo et al. 2004; Zuo \& Lombardo 2010; Gao et al. 2011; Tanigawa et al. 2004; Kaminker et al. 2002; Xu et al. 2013, 2012a; Tang et al. 2013; Liu \& Wang 2013). Neutrons, protons in the interior of NSs can become the superfluid states due to the attraction between two neutrons or protons. Neutrons in NS crust probably form ${ }^{1} S_{0}$ pairing and in NS core mainly form ${ }^{3} P_{2}$ pairing. Protons in NS core can suffer ${ }^{1} S_{0}$ pairing which appear in NS matter with supranuclear density, such density region is related largely to the direct Urca processes on nucleons(Yakovlev et al. 1999; Shternin et al. 2011; Chen et al. 2006). And it is well known that the direct Urca processes on nucleons produce the most powerful neutrino energy losses(Haensel \& Gnedin 1994; Gnedin et al. 1994; Yakovlev et al. 2008; Xu et al. 2014). Thus nucleonic superfluids must affect NS cooling.

The ${ }^{1} S_{0}$ nucleonic pairing gap has been considered using different model potentials of the nucleonnucleon(NN) interaction. These theoretic calculations based on qualitative models give similar ranges 
for the presence of the ${ }^{1} S_{0}$ nucleonic pairing. Nevertheless, due to many uncertain factors about the NN interaction such as the non-direct observational data in extreme conditions, approximations used in the calculations, it can not get the accurate results for the pairing gap and estimate the superfluid quantitative influence on NSs. Nowdays, many of relativistic models are drawing attention in studies on NSs because they are particularly suited for describing NSs according to the special relativity. The most common among them is the relativistic mean field(RMF) theory which could give very successful descriptions in nuclear matter and finite nuclei researches (Glendenning 1985). In 1993, to adapt to the effective $\Lambda \Lambda$ interaction $\Delta B_{\Lambda \Lambda} \sim 5 \mathrm{MeV}$ inferred from the earlier measurement and avoid the high-density instability in numerical calculation, Schaffner et al extended the standard RMF model by adding strange mesons $\sigma^{*}$ and $\phi$ (Schaffner et al. 1993). That is, baryons interact by exchanging the $\sigma, \omega, \rho, \sigma^{*}$ and $\phi$ mesons. While the recent measurement suggests that $\Delta B_{\Lambda \Lambda}$ should be $1.01 \pm 0.20_{-0.11}^{+0.18} \mathrm{MeV}$ (Bednarek \& Manka 2005; Yang \& Shen 2008; Wang \& Shen 2010; Xu et al. 2012b). In addition, when baryonic density is lower than $2 \rho_{0}$ ( $\rho_{0}$ is the saturation density of nuclear matter), NSs are generally made up of only neutrons, protons and leptons. Yet if baryonic density exceeds about $2 \rho_{0}$, hyperons as new degrees of freedom will appear in NSs cores. They must result in the equation of state(EOS) changing, such the nucleonic Fermi momenta and single particle energies tend to change. So the hyperonic appearance would affect the nucleonic SF.

The content of the paper is arranged in this way. The NS properties and ${ }^{1} S_{0}$ nucleonic pairing gap are described using RMF and Bardeen-Cooper-Schrieffer(BCS) theories in Sec.2. The numerical results are displayed in Sec.3. The summary is presented in Sec.4. $\Sigma$ hyperons are ruled out due to the debatable $\Sigma$ potential at $\rho_{0}$ in nuclear matter (Batty et al. 1994). The paper is primarily focused upon the influence of hyperons on the ${ }^{1} S_{0}$ nucleonic pairing gap.

\section{THE MODELS}

Baryonic interactions are by exchanging of $\sigma, \omega, \rho, \sigma^{*}, \phi$ mesons in RMF approach. In this paper, $\mathrm{n}, \mathrm{p}$, $\Lambda$ and $\Xi$ baryons are considered in NSs. The contribution of the first three mesons to the lagrangian is (Glendenning 1985),

$$
\begin{gathered}
L=\sum_{B} \bar{\psi}_{B}\left[i \gamma_{\mu} \partial^{\mu}-\left(M_{B}-g_{\sigma B} \sigma\right)-g_{\rho B} \gamma_{\mu} \boldsymbol{\tau} \cdot \boldsymbol{\rho}^{\mu}-g_{\omega B} \gamma_{\mu} \omega^{\mu}\right] \psi_{B}+\frac{1}{2}\left(\partial_{\mu} \sigma \partial^{\mu} \sigma-m_{\sigma}^{2} \sigma^{2}\right)-U(\sigma)+\frac{1}{2} m_{\omega}^{2} \omega_{\mu} \omega^{\mu} \\
+\frac{1}{4} c_{3}\left(\omega_{\mu} \omega^{\mu}\right)^{2}+\frac{1}{2} m_{\rho}^{2} \boldsymbol{\rho}_{\mu} \boldsymbol{\rho}^{\mu}-\frac{1}{4} F^{\mu v} F_{\mu v}-\frac{1}{4} G^{\mu v} G_{\mu v}+\sum_{l} \bar{\psi}_{l}\left[i \gamma_{\mu} \partial^{\mu}-m_{l}\right] \psi_{l}
\end{gathered}
$$

Where the field tensors of the vector mesons $\omega$ and $\rho$ are marked as $F_{\mu v}$ and $G_{\mu v}$, respectively. $U(\sigma)=$ $\frac{1}{3} a \sigma^{3}+\frac{1}{4} b \sigma^{4}$. The baryon species are marked as B. The contribution of strange mesons $\sigma^{*}, \phi$ to the lagrangian is,

$$
L^{Y Y}=\frac{1}{2}\left(\partial_{v} \sigma^{*} \partial^{v} \sigma^{*}-m_{\sigma^{*}}^{2} \sigma^{* 2}\right)-\sum_{B} g_{\sigma^{*} B} \bar{\psi}_{B} \psi_{B} \sigma^{*}-\sum_{B} g_{\phi B} \bar{\psi}_{B} \gamma_{\mu} \psi_{B} \phi^{\mu}-\frac{1}{4} S^{\mu v} S_{\mu v}+\frac{1}{2} m_{\phi}^{2} \phi_{\mu} \phi^{\mu}
$$

Here $\sigma^{*}, \phi$ aren't coupled with nucleons, they only affect the hyperonic properties.

The five meson fields are considered as classical fields, and the field operators are replaced with their expectation values in RMF model(Glendenning 1985; Bednarek \& Manka 2005; Yang \& Shen 
2008; Wang \& Shen 2010; Xu et al. 2012b). The meson field equations in NSs are as follows:

$$
\begin{array}{r}
\sum_{B} g_{\sigma B} \rho_{S B}=m_{\sigma}^{2} \sigma+a \sigma^{2}+b \sigma^{3}, \\
\sum_{B} g_{\omega B} \rho_{B}=m_{\omega}^{2} \omega_{0}, \\
\sum_{B} g_{\rho B} \rho_{B} I_{3 B}=m_{\rho}^{2} \rho_{0}, \\
\sum_{B} g_{\sigma^{*} B} \rho_{S B}=m_{\sigma^{*}}^{2} \sigma^{*}, \\
\sum_{B} g_{\phi B} \rho_{B}=m_{\phi}^{2} \phi_{0} .
\end{array}
$$

Here $I_{3 B}$ is the isospin projections of baryon species B. $\rho_{S B}$ and $\rho_{B}$ mark baryonic scalar and vector densities, respectively. They are,

$$
\begin{array}{r}
\rho_{S B}=\frac{1}{\pi^{2}} \int_{0}^{k_{F}} \frac{m_{B}^{*}}{\sqrt{k^{2}+m_{B}^{* 2}}} k^{2} d k, \\
\rho_{B}=\frac{k_{F}^{3}}{3 \pi^{2}} .
\end{array}
$$

Here $k_{F}$ is the baryonic Fermi momentum, $m_{B}^{*}=m_{B}-g_{\sigma B} \sigma_{0}-g_{\sigma^{*} B} \sigma_{0}^{*}$ is the baryonic effective mass.

A description of NS matter with uniform distribution is obtained through the conditions of electrical neutrality and $\beta$ equilibrium. The electrical neutrality condition is

$$
\rho_{p}=\rho_{\Xi^{-}}+\rho_{e}+\rho_{\mu}
$$

The baryonic chemical potential is expressed by

$$
\mu_{B}=\mu_{n}-q_{B} \mu_{e}
$$

where $q_{B}$ is the baryonic electric charge number. Then the $\beta$ equilibrium conditions are given by

$$
\begin{array}{r}
\mu_{n}=\mu_{p}+\mu_{e}, \quad \mu_{\Xi^{-}}=\mu_{n}+\mu_{e} \\
\mu_{n}=\mu_{\Lambda}=\mu_{\Xi^{0}}, \quad \mu_{e}=\mu_{\mu} .
\end{array}
$$

The nucleonic single-particle energy in the model is

$$
E_{N}(k)=\sqrt{k^{2}+m_{N}^{*}}+g_{\omega N} \omega_{0}+g_{\rho N} \rho_{03} I_{3 N} .
$$

The BCS gap equation is(Zuo et al. 2004; Chen et al. 2006; Xu et al. 2013),

$$
\Delta_{N}(k)=-\int \frac{V_{N N}\left(k, k^{\prime}\right) \Delta_{N}\left(k^{\prime}\right) k^{\prime 2} d k^{\prime}}{4 \pi^{2} \sqrt{\left[\left(E_{N}\left(k^{\prime}\right)-E_{N}\left(k_{F}\right)\right]^{2}+\Delta_{N}^{2}\left(k^{\prime}\right)\right.}} .
$$

The ${ }^{1} S_{0}$ pairing gaps of neutrons and protons are calculated based on the Reid soft-core(RSC) interaction(Nishizaki et al. 1991; Sprung \& Banerjee 1971; Amundsen \& Østgaard 1985; Wambach et al. 1993), The ${ }^{1} S_{0}$ channel interaction between two neutrons or protons is

$$
V_{N N}\left(k, k^{\prime}\right)=4 \pi \int r^{2} d r j_{0}(k r) V_{N N}(r) j_{0}\left(k^{\prime} r\right)
$$


Table 1 The TM1 set, the masses are in unit of MeV (Yang \& Shen 2008)

\begin{tabular}{ccccccccccc}
\hline$m_{\sigma}$ & $g_{\sigma N}$ & $m_{\omega}$ & $g_{\omega N}$ & $m_{\rho}$ & $g_{\rho N}$ & $c_{3}$ & $g_{2}\left(\mathrm{fm}^{-1}\right)$ & $g_{3}$ & $m_{N}$ & $m_{\phi}$ \\
\hline 511.198 & 10.029 & 783.0 & 12.614 & 770.0 & 4.632 & 71.308 & 7.233 & 0.618 & 983.0 & 1020.0 \\
\hline
\end{tabular}

Table 2 The scalar coupling constants of hyperons, the masses are in unit of $\mathrm{MeV}$

\begin{tabular}{rccccc}
\hline$m_{\sigma^{*}}$ & $m_{\phi^{*}}$ & $g_{\sigma \Lambda}$ & $g_{\sigma \Xi}$ & $g_{\sigma^{*} \Lambda}$ & $g_{\sigma^{*} \Xi}$ \\
\hline with $\sigma^{*} \phi 975.0$ & 1020.0 & 6.170 & 3.202 & 5.412 & 11.516 \\
\hline
\end{tabular}

Table 3 Baryonic density ranges for the ${ }^{1} S_{0}$ nucleonic pairing gap $\Delta_{N}\left(k_{F}\right)$ at the Fermi surface in npe $\mu$ and npHe $\mu$ matter

\begin{tabular}{cc}
\hline nре $\mu$ & $\mathrm{npHe} \mu$ \\
\hline$\Delta_{n}\left(k_{F}\right) 0.0 \leq \rho_{B} \leq 0.188$ & $0.0 \leq \rho_{B} \leq 0.191$ \\
$\Delta_{p}\left(k_{F}\right) 0.0 \leq \rho_{B} \leq 0.5090 .0 \leq \rho_{B} \leq 0.588$ \\
\hline
\end{tabular}

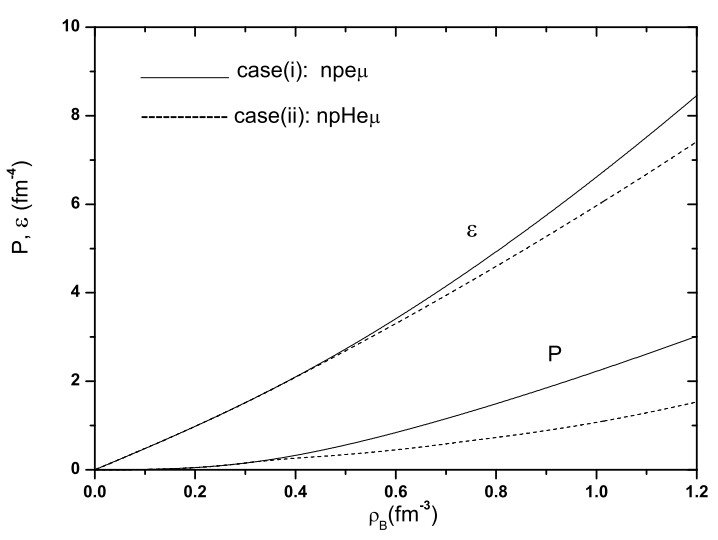

Fig. 1 Pressure $\mathrm{P}$ and energy density $\varepsilon$ as functions of baryonic density $\rho_{B}$ in npe $\mu$ and npHe $\mu$ matter.

where $V_{N N}(r)$ is the ${ }^{1} S_{0} \mathrm{NN}$ interaction potential in coordinate space, $j_{0}(k r)$ is the zero order spherical Bessel function.

The nucleonic critical temperature $T_{C N}$ of the ${ }^{1} S_{0}$ pairing SF is(Takatsuka \& Tamagaki 2003),

$$
T_{C N} \doteq 0.66 \Delta_{N}\left(k_{F}\right) \times 10^{10} .
$$

According to the discussion of the RMF approach above, we can obtain the EOS and NS composition as well as the nucleonic Fermi momenta and single particle energies which are vitally important in the research of the $\mathrm{NN}$ pairing gap. 


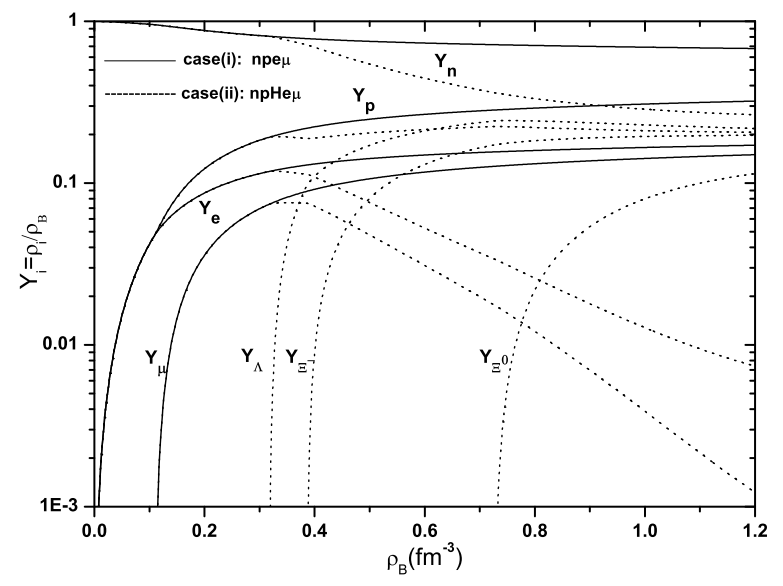

Fig. 2 Composition of NSs as functions of baryonic density $\rho_{B}$.

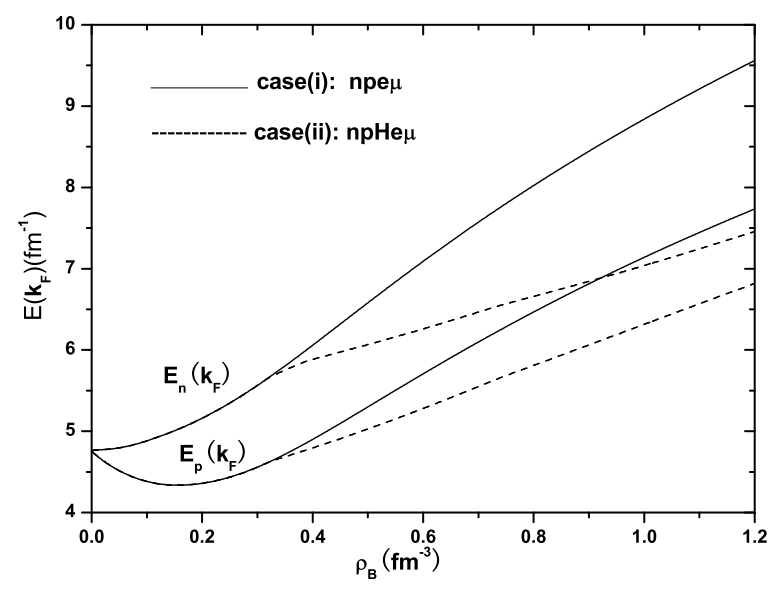

Fig. 3 The nucleonic single particle energy $E_{N}\left(k_{F}\right)$ at the Fermi surface vs baryonic density $\rho_{B}$ in npe $\mu$ and npHe $\mu$ matter.

\section{DISCUSSION}

According to the uncertainty of interior constitution of NSs, we research NSs in both cases: (i) NS copnsition is n, p, e, $\mu($ npe $\mu)$, (ii) n, p, $\Lambda, \Xi^{0}, \Xi^{-}$, e, $\mu(\operatorname{npHe} \mu)$. This work focuses on the influence of hyperons on the ${ }^{1} S_{0}$ nucleonic pairing gap in NSs. The appearance of hyperons changes the EOS and NS composition as well as the ${ }^{1} S_{0}$ nucleonic pairing SF. It is widely accepted that the ${ }^{1} S_{0}$ nucleonic superfluids should be decided by pairing gap $\Delta_{N}(k)$. Next, we'll show the numerical results for the 


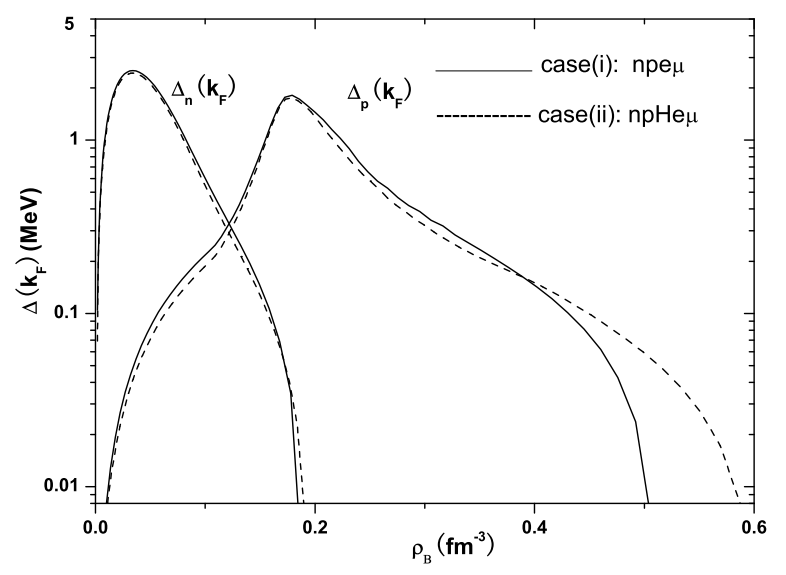

Fig. 4 The ${ }^{1} S_{0}$ nucleonic pairing gap $\Delta_{N}\left(k_{F}\right)$ at the Fermi surface as functions of baryonic density $\rho_{B}$ in npe $\mu$ and npHe $\mu$ matter.

${ }^{1} S_{0}$ nucleonic pairing gaps in npe $\mu$ and npHe $\mu$ matter. The NSs' properties are getted using a set of parameters displayed in Table 1,2. We use $U_{\Lambda}^{N}=-30 \mathrm{MeV}, U_{\Sigma}^{N}=+30 \mathrm{MeV}, U_{\Xi}^{N}=-18 \mathrm{MeV}$, and $U_{\Lambda}^{\Lambda}=-5 \mathrm{MeV}$ which are obtained based upon the recent measurement $\Delta B_{\Lambda \Lambda} \sim 1.01 \pm 0.20_{-0.11}^{+0.18} \mathrm{MeV}$ to decide the hyperonic scalar coupling constants. We use $\frac{2}{3} g_{\omega N}=g_{\omega \Lambda}=2 g_{\omega \Xi}, g_{\rho N}=g_{\rho \Xi}, g_{\rho \Lambda}=0$, $2 g_{\phi \Lambda}=g_{\phi \Xi}=-\frac{2 \sqrt{2}}{3} g_{\omega N}$ to calculate the hyperonic vector coupling constants (Bednarek \& Manka 2005; Yang \& Shen 2008; Wang \& Shen 2010; Xu et al. 2012b).

As mentioned above, baryons interact by exchanging mesons. More specifically, the attraction, repulsion and isospin interaction between two baryons are supplied by $\sigma, \omega$ and $\rho$, respectively. And the additional attraction and repulsion between two hyperons are supplied by strange mesons $\sigma^{*}$ and $\phi$, respectively. Fig. 1 gives the EOS, namely, pressure $P$ and energy density $\varepsilon$ as functions of baryonic density $\rho_{B}$ in npe $\mu$ and npHe $\mu$ matter. As shown in Fig.1, one could see that the pressure $P$ and energy density $\varepsilon$ remain unchanged at lower densities in both cases. While with increasing of baryonic density, the appearance of hyperons makes the pressure $P$ and energy density $\varepsilon$ decrescent. That is, the EOS is softened which will inevitably cause the NS's bulk property changing. The crucial physical quantities for the ${ }^{1} S_{0}$ nucleonic pairing gap are the nucleonic Fermi momenta and single-particle energies. Fig.2 shows the numerical results of NS composition as functions of baryonic density $\rho_{B}$ in npe $\mu$ and npHe $\mu$ matter. As shown in Fig.2, the threshold densities of $\Lambda, \Xi^{-}$and $\Xi^{0}$ hyperons are $0.320 \mathrm{fm}^{-3}$, $0.389 \mathrm{fm}^{-3}$ and $0.734 \mathrm{fm}^{-3}$, respectiely. One can also see that nucleonic fractions are suppressed due to hyperons appearing in NSs through the conditions of electrical neutrality and $\beta$ equilibrium(see Eqs.911). Therefore, according to Eq.8, we can see that when hyperons appear in NSs, the individual Fermi momenta of neutrons and protons are all much less than their values in npe $\mu$ matter. Fig. 3 displays the nucleonic single particle energy $E_{N}\left(k_{F}\right)$ at the Fermi surface as functions of baryonic density $\rho_{B}$ in nре $\mu$ and npHe $\mu$ matter. As Fig. 3 shows, the nucleonic single particle energies in npHe $\mu$ matter are obviously less than their values in npe $\mu$ matter which is because the reduction of the nucleonic Fermi momenta resulting in $E_{n}\left(k_{F}\right)$ and $E_{p}\left(k_{F}\right)$ all decreasing in npHe $\mu$ matter(see Eq.12).

So far, due to the uncertainty of $\mathrm{NN}$ interaction, the ${ }^{1} S_{0}$ nucleonic pairing gap is also uncertain. In the work, we calculate $\Delta_{N}(k)$ based on the RSC potential. The main concentration of us is the hyperonic influences on the ${ }^{1} S_{0}$ nucleonic pairing gap. Fig. 4 presents the ${ }^{1} S_{0}$ nucleonic pairing gap at the Fermi 
surface as functions of baryonic density $\rho_{B}$ in npe $\mu$ and npHe $\mu$ matter. In Fig.4, one can see that the ${ }^{1} S_{0}$ neutronic pairing gap always exists in lower densities region in both cases. The region of neutronic superfluids only affects the NS's surface cooling. The ${ }^{1} S_{0}$ protonic superfluids can reach relatively high densities. The region of protonic superfluids is closely related to the direct Urca processes on nucleons which governs almost the cooling processes of NSs. In addition, one can also see that the appearance of hyperons has little impact on baryonic density range and size for the ${ }^{1} S_{0}$ neutronic pairing gap $\Delta_{n}\left(k_{F}\right)$ in Fig.4. This is because the ${ }^{1} S_{0}$ neutronic superfluids appear only in the region of lower densities where hyperons have not appeared in NS matter. To see clearly the influence of hyperons degrees of freedom on the ${ }^{1} S_{0}$ nucleonic pairing gap more intuitively, baryonic density ranges for the ${ }^{1} S_{0}$ nucleonic pairing gap $\Delta_{N}\left(k_{F}\right)$ at the Fermi surface in npe $\mu$ and npHe $\mu$ matter are listed in Table 3. As seen in Fig.4 and Table 3 , when hyperons appear in NS matter, the ${ }^{1} S_{0}$ protonic pairing gap $\Delta_{p}\left(k_{F}\right)$ decreases slightly in the region of $\rho_{B}=0.0-0.393 \mathrm{fm}^{-3}$, and increases obviously in the region of $\rho_{B}=0.393-0.588 \mathrm{fm}^{-3}$ which is because the appearance of $\Lambda$ and $\Xi^{-}$hyperons in NS core(see Fig. 2 for details). The increase of the ${ }^{1} S_{0}$ protonic pairing gap must lead to the increase of the protonic critical temperature $T_{C P}$ (see Eq.15), then the neutrino energy losses of the direct Urca on nucleon would be further suppressed in the region of $\rho_{B}=0.393-0.588 \mathrm{fm}^{-3}$. And the range of the ${ }^{1} S_{0}$ protonic SF is obviously enlarged due to the presence of hyperons, which can achieve coverage or partial coverage NSs cores. That is, If we don't consider the contributions of the direct Urca processes on hyperons on NS cooling, the presence of hyperons must decrease the cooling rate of NSs.

\section{CONCLUSION}

We study the effects of hyperons on the ${ }^{1} S_{0}$ nucleonic SF by adopting the RMF and BCS theories in NSs. The results indicate that the appearance of hyperons has little influence on baryonic density range and size for the ${ }^{1} S_{0}$ neutronic SF. However, the ${ }^{1} S_{0}$ protonic pairing gap (and the ${ }^{1} S_{0}$ protonic critical temperature) in npHe $\mu$ matter is much larger than their values in npe $\mu$ matter in the region of $\rho_{B}=0.393-0.588 \mathrm{fm}^{-3}$. The baryonic density range of the ${ }^{1} S_{0}$ protonic SF is also enlarged from $\rho_{B}=0.0-0.509 \mathrm{fm}^{-3}$ to $\rho_{B}=0.0-0.588 \mathrm{fm}^{-3}$ on account of the presence of hyperons. The changes of the ${ }^{1} S_{0}$ protonic SF can further suppress the NS's cooling rate. And hyperons in NSs change the ${ }^{1} S_{0}$ protonic pairing gap which must affect the cooling properties of NSs.

Our model may simplify because it adopts the lowest level of approximation in the BCS equation as well as neglects the possible influence of the inhomogeneous in NS crust and ${ }^{1} S_{0}$ hyperonic pairing in NS core on the ${ }^{1} S_{0}$ nucleonic energy gap. While it can give the clear influence of hyperons degrees of freedom on the ${ }^{1} S_{0}$ nucleonic pairing. We will analyze more complicated models in future studies.

Acknowledgements This work is funded by the National Natural Science Foundation of China under grant Nos.11373047, 11103047 and 11303063.

\section{References}

Amundsen, L., \& Østgaard, E. 1985, Nucl Phys A, 437, 4873

Batty, C. J., Friedman, E., \& Gal, A. 1994, Phys Lett B, 335, 2732

Bednarek, I., \& Manka, R. 2005, J Phys G: Nucl Part Phys, 31, 1009 2, 6

Chen, W., Lam, Y. Y., \& Wen, D. H. 2006, Chin Phys Lett, 23, 271 1, 3

Gao, Z. F., Peng, Q. H., Wang, N., Chou, C. K., \& Huo, W. S. 2011, Ap\&SS, 336, 4271

Glendenning, N. K. 1985, ApJ, 293, 4702

Gnedin, O. Y., Yakovlev, D. G., \& Shibanov, Y. A. 1994, Astron lett, 20, 4091

Haensel, P., \& Gnedin, O. Y. 1994, A\&A, 290, 4581

Kaminker, A. D., Yakovlev, D. G., \& Gnedin, O. Y. 2002, A\&A, 383, 10761

Liu, M.-Q., \& Wang, Z.-X. 2013, Res Astron Astrophys, 13, 2071

Nishizaki, S., Takatsuka, T., Yahagi, N., \& Hiura, J. 1991, Prog Theor Phys, 86, 8533

Schaffner, J., Dover, C. B., Gal, A., Greiner, C., \& Stöcker, H. 1993, Phys Lett B, 71, 13282 
Shternin, P. S., Yakovlev, D. G., Heinke, C. O., Ho, W. C. G., \& Patnaude, D. J. 2011, MNRAS, 412, L108 1

Sprung, D. W. L., \& Banerjee, P. K. 1971, Nucl Phys A, 168, 2733

Takatsuka, T., \& Tamagaki, R. 2003, Nucl Phys A, 721, 10034

Tang, Y.-Y., Dai, Z.-C., \& Zhang, L. 2013, Res Astron Astrophys, 13, 5371

Tanigawa, T., Matsuzaki, M., \& Chiba, S. 2004, Phys. Rev. C, 70, 0658011

Wambach, J., Ainsworth, T. L., \& Pines, D. 1993, Nucl Phys A, 555, 1283

Wang, Y. N., \& Shen, H. 2010, Phys. Rev. C, 81, 025801 2, 3, 6

Xu, Y., Liu, G. Z., Liu, C. Z., et al. 2013, Chin Phys Lett, 30, 062101 1, 3

Xu, Y., Liu, G. Z., Liu, C. Z., et al. 2014, Chin Sci Bull, 59, 2731

Xu, Y., Liu, G. Z., Wang, H. Y., Ding, W. B., \& Zhao, E. G. 2012a, Chin Phys Lett, 29, 0597011

Xu, Y., Liu, G. Z., Wu, Y. R., et al. 2012b, Plasma Sci Technol, 14, 375 2, 3, 6

Yakovlev, D. G., Gnedin, O. Y., Kaminker, A. D., \& Potekhin, A. Y. 2008, in AIP Conf Proc, vol. 983, 379-387 1

Yakovlev, D. G., Levenfish, K. P., \& Shibanov, Y. A. 1999, Phys Uspek, 42, 7371

Yang, F., \& Shen, H. 2008, Phys. Rev. C, 77, 025801 2, 4, 6

Zuo, W., Li, Z. H., Lu, G. C., et al. 2004, Phys Lett B, 595, 44 1, 3

Zuo, W., \& Lombardo, U. 2010, in AIP Conf Proc, vol. 1235, 235-241 1 